\title{
The impact of surface coverage on the kinetics of electron transfer through redox monolayers on a silicon electrode surface
}

\begin{abstract}
The impact of the coverage of ferrocene moieties, attached to a silicon electrode modified via hydrosilylation of a dialkyne, on the kinetics of electron transfer between the redox species and the electrode is explored. The coverage of ferrocene is controlled by varying the coupling time between azidomethyl ferrocene and the distal alkyne of the monolayer via the copper assisted azide-alkyne cyclo addition reaction. All other variables in the surface preparation are maintained identical. What is observed is that the higher the surface coverage of the ferrocene moieties the faster the apparent rates of electron transfer. This surface coveragedependent kinetic effect is attributed to electrons hopping between ferrocene moieties across the redox film toward hotspots for the electron transfer event. The origin of these hotspots is tentatively suggested to result from minor amounts of oxide on the underlying silicon surface that reduce the barrier for the electron transfer.
\end{abstract}

Keyword: Hydrosilylation; Si(100) monolayers; Electrode kinetics 